\begin{tabular}{|c|c|}
\hline Title & Role of Nitrogen Precursor Supplies on InA s Quantum Dot Surfaces in Their Emission Wavelengths \\
\hline Author(s) & $\begin{array}{l}\text { Suemune, Ikuo; Sasikala, Ganapathy; Kumano, Hidekazu; U esugi, Katsuhiro; Nabetani, Y oichi; Matsumoto, Takashi; } \\
\text { Maeng, J.-T.; Seong, T ae Y eon }\end{array}$ \\
\hline Citation & $\begin{array}{l}\text { Japanese Journal of A pplied Physics, 45(no. 21), L529-L532 } \\
\text { https://doi.org/10.1143/JJA P.45.L529 }\end{array}$ \\
\hline Issue Date & 2006 \\
\hline Doc URL & http://hdl.handle.net/2115/33910 \\
\hline Type & article (author version) \\
\hline File Information & Nitrogen purge paper-JJA P.pdf \\
\hline
\end{tabular}

Instructions for use 


\section{Role of Nitrogen Precursor Supplies on InAs Quantum Dot Surfaces in Their Emission Wavelengths}

Ikuo SUEMUNE, Ganapathy SASIKALA, Hidekazu KUMANO, Katsuhiro UESUGI, Yoichi NABETANI $^{1}$, Takashi MATSUMOTO ${ }^{1}$, J.-T. MAENG ${ }^{2}$ and Tae Yeon SEONG ${ }^{2}$

Research Institute for Electronic Science, Hokkaido University, Souseitou, Kita 21, Nishi 10, Sapporo 001-0021, Japan and CREST, Japan Science and Technology Corporation, Japan

${ }^{1}$ Interdisciplinary Graduate School of Medicine and Engineering, University of Yamanashi, Takeda 4-3-11, Kofu 400-8511, Japan

${ }^{2}$ Department of Materials Science and Engineering, Gwangju Institute of Science and Technology, 1 Oryong-dong, Puk-gu, Gwangju 500-712, Korea

The role of nitrogen $(\mathrm{N})$ in emission wavelengths from InAs quantum dots (QDs) was studied with intentional supplies of a $\mathrm{N}$ precursor (monomethyl hydrazine) to InAs dot surfaces just before burying them with GaAs capping layers by metalorganic molecular-beam epitaxy. Luminescence from the InAs QDs showed a red shift with the N-precursor supplies on the dot surfaces, and more clear separations of the luminescence sub peaks originating from QD excited-state transitions were observed. The increase in the $\mathrm{N}$ precursor supplies on the dot surfaces however changed the relative variation in the emission wavelengths from the red-shift to the blue shift. Additional observations of the dot sizes as well as the model calculations of the emission peaks and of the energy separations of the lowest and QD excited-state transitions showed that the red-shift is due to the reduced Ga inclusion in the InAs QDs and that the blue shift is due to the reduced dot sizes.

KEYWORDS: quantum dot, nitrogen, monomethylhydrazine, InAs, nitridation 
The extension of emission wavelengths of InAs quantum dots (QDs) to $1.55 \mu \mathrm{m}$ has been extensively studied to realize QD lasers for optical-fiber communications and single-photon emitters for single-photon-based quantum cryptography. ${ }^{1,2)}$ Although InAs QDs grown on GaAs substrates are more difficult to realize with longer emission wavelengths compared with those grown on InP substrates, ${ }^{3,4)}$ the former is advantageous in term of cavity formation based on $\mathrm{GaAs} / \mathrm{Al}(\mathrm{Ga}) \mathrm{As}$ high-reflection distributed Bragg mirrors, which enhances photon extraction efficiencies from QDs. ${ }^{5)}$ The main reason why the extension of the emission wavelengths to a longer wavelength range is difficult stems from the excessive in-plane compressive strain induced within the InAs QDs grown on GaAs substrates due to a large lattice mismatch of $7.2 \%,{ }^{6)}$ which expands the InAs energy gap.

One approach to solve this issue is the use of GaInAs instead of GaAs to bury InAs QDs to minimize the GaInAs/InAs interface lattice mismatch. ${ }^{7,8)}$ However this method in principle will accumulate total strain in the system with an increase in the stacked QD layers and this excess strain accumulation can cause degradations of the QD optical qualities. Another approach is to compensate the compressive strain in InAs QDs by burying them with tensile-strained GaAsN layers, ${ }^{9)}$ and this method demonstrated the systematic extension of the InAs QD emission wavelengths up to $\sim 1.55 \mu \mathrm{m}$ with an increase in the nitrogen $(\mathrm{N})$ concentrations to $2.7 \% .^{10)}$ This method to bury InAs QDs with the GaAsN strain compensating layer (SCL) has an advantage in that the overall strain is compensated and the accumulation of the total strain in the system can be minimized, which increases luminescence efficiencies up to fivefold compared with QDs buried conventionally with GaAs layers. ${ }^{11)}$

However, in the discussion of the mechanism responsible for the red-shift of the emission wavelength in the latter case, there still remains the possibility that $\mathrm{N}$ may diffuse from the GaAsN burying layers into the InAs QDs. The formation of $\operatorname{In}(\mathrm{Ga}) \mathrm{AsN}$ dots can red-shift the emission wavelength. ${ }^{12)}$ If the two factors, i.e., the strain compensation of InAs QDs with the GaAsN SCLs and the $\mathrm{N}$ diffusion effect from the GaAsN layers into the buried InAs QDs, are well separated, clearer discussions on the role of $\mathrm{N}$ in the observed red-shifts will be possible.

In this study, InAs QDs were buried with GaAs layers, whereas InAs dot surfaces were intentionally exposed to $\mathrm{N}$-precursor supplies before the burying processes. The intentional supplies of a $\mathrm{N}$-precursor to the InAs dots surfaces will be an extreme case to examine the possibility of $\mathrm{N}$ diffusion into the InAs QDs. It is shown that the red-shifts observed with this "nitridation" process is limited to a wavelength of $1150 \mathrm{~nm}$ and a blue shift rather than a red-shift is observed with a prolonged "nitridation". These results will be studied with the observations of dot sizes by atomic force microscopy (AFM) and transmission electron 
microscopy (TEM). The "nitridation” effect will be discussed with a QD model calculation.

InAs QD samples were grown on GaAs (001) substrates using metalorganic molecular-beam epitaxy (MOMBE). The metalorganic precursors used were triethylgallium (TEGa), triethylindium (TEIn), trisdimethylaminoarsenic (TDMAAs), and monomethylhydrazine (MMHy) for gallium, indium, arsenic, and nitrogen, respectively. After oxide desorption from a GaAs substrate surface, a 100-nm-thick GaAs buffer layer was grown at $550{ }^{\circ} \mathrm{C}$ with the respective beam equivalent pressures (BEP) of $8 \times 10^{-4}$ and $30 \times 10^{-4} \mathrm{~Pa}$ for TEGa and TDMAAs. The substrate temperature was then lowered to $400{ }^{\circ} \mathrm{C}$, and approximately 2.0 monolayers (ML) of InAs were deposited with a pulsed supply of TEIn with a BEP of $0.8 \times 10^{-4}$ $\mathrm{Pa}$ at a medium growth rate of $0.1 \mathrm{ML} / \mathrm{s}$. TDMAAs are continuously supplied during the growth of InAs. A transition from the two-dimensional to three-dimensional growth modes, i.e., the initiation of the Stranski-Krastanow growth mode, was confirmed using reflection high-energy electron diffraction (RHEED) analysis by transformation from streaky to spotty patterns.

After the QD growth, MMHy was supplied to the InAs QD surfaces at $400{ }^{\circ} \mathrm{C}$, where different BEPs of MMHy are supplied depending on the samples. In the previous studies of InAs QDs buried with GaAsN layers, GaAsN alloys with $\mathrm{N}$ concentrations up to $3 \%$ were grown at $400^{\circ} \mathrm{C}$ using MMHy. ${ }^{10}$ ) The sequence of the MMHy supply in this study is as follows: MMHy was supplied to InAs QDs with a BEP range of $1.5 \times 10^{-4} \sim 15 \times 10^{-4} \mathrm{~Pa}$ for $30 \mathrm{~s}$ and then the MMHy supply was turned off and was purged for $30 \mathrm{~s}$ with the simultaneous supply of TDMAAs to prevent As desorption. This sequence will be called "nitridation" process hereafter and was repeated 5 times for all the "nitridation" samples.

After this "nitridation" process, a 20-nm-thick GaAs capping layer was grown to bury the InAs QDs. The same growth procedure except for the MMHy supply sequence was used for the growth of the reference InAs QD sample without the "nitridation” process. The grown QD structures were studied using AFM and TEM, and photoluminescence (PL) measurements were carried out by exciting the samples using the second-harmonic generation of a YAG laser at a wavelength of $532 \mathrm{~nm}$. The measurements were performed between $20 \mathrm{~K}$ and room temperature.

Figure 1(a) shows the PL spectra measured at $20 \mathrm{~K}$ of the InAs QD samples. The reference InAs QD sample grown without “nitridation” showed a PL peak at $975 \mathrm{~nm}$. With "nitridation", the PL peak showed a red shift with an increase in the MMHy BEP up to $4 \times 10^{-4} \mathrm{~Pa}$. However, further increase in the MMHy BEP induced a relative blue-shift of the PL peak. The MMHy BEP dependence of the PL peaks is summarized in Fig. 1(b). The red-shift in the lower 
MMHy BEP range is substantial and the subsequent relative blue-shift in the higher MMHy BEP range shows a more gradual dependence on the MMHy BEP. This suggests the presence of at least two different mechanisms to control PL peak shifts during the "nitridation" process. The PL peaks also showed splitting into sub peaks due to the QD excited-state transitions. The energy separations of the dominant lower two sub peaks were estimated by fitting the PL spectra with multiple Gaussian lineshapes ${ }^{9)}$ and showed a monotonous increase for the increase in the MMHy BEP as shown in Fig. 1(a). The observed dependence of the PL peaks on the "nitridation" processes will be discussed later with the additional observations of QD structures.

The influence of the MMHy supply on the InAs QD surfaces was examined by AFM measurements. As-grown InAs QDs showed a dot density of $1.4 \times 10^{11} \mathrm{~cm}^{-2}$ as shown in Fig. 2(a). Figure 2(b) shows an AFM image of the QD sample after "nitridation" with an MMHy BEP of $4 \times 10^{-4} \mathrm{~Pa}$. The increase in the dot sizes and the reduction in the dot density to $\sim 1 / 3$ are clearly observed. Similar dot size and shape modifications have been reported during the burying process of QDs with $\mathrm{GaAs}^{13)}$ and AlGaAs. ${ }^{14)}$

Details of the dot size distributions are summarized in Fig. 3. The relations of the QD heights and widths are shown as closed circles and open squares, respectively, and open circles for the reference sample without "nitridation" and the ones with "nitridation" with an MMHy BEP of $4 \times 10^{-4} \mathrm{~Pa}$ and $15 \times 10^{-4} \mathrm{~Pa}$, respectively. The average QD lateral sizes $\left(\mathrm{W}_{\mathrm{av}}\right)$ and heights $\left(\mathrm{H}_{\mathrm{av}}\right)$ are also shown in Fig. 3 with the stars which are located around the middle of each group. The QD size distributions clearly show the dependence on "nitridation", and the dot heights increased once with an increase in the MMHy BEP during the "nitridation" process but were reduced with the further increase in the MMHy BEP.

Some of the InAs QDs were also examined by cross-sectional TEM observations. A cross-sectional TEM image of the reference InAs QDs without "nitridation" showed the height $H$ and the lateral size $W$ of the QD as 2.9 and $9.7 \mathrm{~nm}$, respectively. Although the number of InAs dots observed by TEM is limited, the main factor for the difference in the observed QD lateral size and the average one measured by AFM may be influenced by an artifact due to AFM canti lever tip-apex curvatures. ${ }^{15)}$ Similar TEM observations of the InAs QDs prepared with

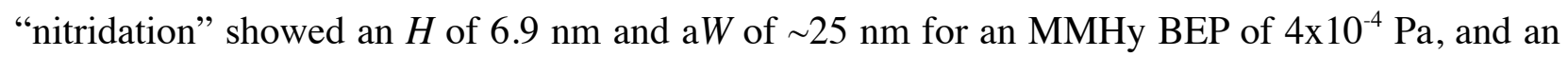
$H$ of $5.0 \mathrm{~nm}$ and $\mathrm{aW}$ of $\sim 20 \mathrm{~nm}$ for $15 \times 10^{-4} \mathrm{~Pa}$. The difference in the dot heights and widths between the TEM observations and the AFM observations shown in Fig. 3 may be due to limited dot numbers examined by the TEM observations and the dot size distributions as shown in Fig. 3.

The origins of the PL peak shifts and the sub peak separations shown in Fig. 1 were examined using a QD model calculation. QDs were approximated using quantum disks with 
harmonic-oscillator-type parabolic potentials to describe lateral confinements. ${ }^{16)}$ Briefly, when the confinement potential in the dot-height direction is sufficiently high enough to confine electrons or holes in the in-plane two-dimensional region, the potential barrier is approximated with the superposition of those in the dot-height direction and in the in-plane radial direction. After the conventional variable separations into the dot-height and in-plane directions, the former results in a one-dimensional quantum confinement problem in the dot-height direction and the latter results in a two-dimensional quantum confinement problem with harmonic oscillator-type potential, which can be solved analytically. ${ }^{16)}$ The energy separation $\hbar \omega$ of its energy states due to the lateral confinement is given by $16 \hbar^{2} /\left(m^{*} W\right)^{2}$, where $\hbar$ is the Planck's constant divided by $2 \pi, \mathrm{m}^{*}$ is the electron or hole effective mass, and $\mathrm{W}$ is the disk diameter.

The main factors included in the model calculation are the following: (1) Ga inclusion into InAs QDs was assumed because a PL peak at $975 \mathrm{~nm}$ cannot be fitted with dot size variations in pure InAs QDs. (2) The strain dependence of the $\operatorname{In}(\mathrm{Ga})$ As energy gap was calculated using the deformation potentials given in ref. 17. Although the strain distributions in real QDs are spatially variant, ${ }^{18)}$ it was assumed to be uniform for simplicity in the present model. (3) InAs and InGaAs are known to have large band nonparabolicity in the conduction band, and energy-dependent effective electron mass was calculated from the $\mathrm{k} \cdot \mathrm{p}$ theory. The other physical parameters necessary for the calculations are available in a published literature. ${ }^{19)}$ Because the valence band offset in InAs/GaAs grown on GaAs is very small $(\sim 12 \mathrm{meV})$ considering the deformation potentials, the quantum confinement effects are predominant in the conduction band.

The calculations were performed for various combinations of the dot height $\mathrm{H}$ and the width $\mathrm{W}$ that will cover the range of the dot sizes observed by AFM and TEM. The results were summarized with the relation between the transition energies of the lowest QD states and the energy separations between the lowest and second QD excited-state transitions. One example calculated for a W/H ratio of 6 is shown in Fig. 4. The nearly horizontal solid lines are calculated by changing the Ga concentrations in InGaAs dots against the constant dot heights $H$ of 3.1, 3.7, 4.8, and $7.1 \mathrm{~nm}$, whereas the crossing solid lines are those calculated by changing $H$ against the constant Ga concentrations of $30,40,50$, and $60 \%$ in the InGaAs dots. The accuracy of the present model calculation was checked by comparing with a pseudopotential calculation for an InAs QD with $H=3.5 \mathrm{~nm}$ and $W=25.2 \mathrm{~nm}$, which resulted in an energy separation of $\sim 80 \mathrm{meV}$ between the first excited state and the lowest excited state. ${ }^{20)}$ The corresponding present model calculation gave energy separations of 72 and $82 \mathrm{meV}$ with the nonparabolic and parabolic electron effective masses, respectively, which are reasonably in agreement with the above pseudopotential calculation. 
The measurements are plotted as closed circles in Fig. $4{ }^{21)}$ The comparison with the model calculation assuming a ratio of $\mathrm{W} / \mathrm{H}=6$ shows that the observed maximum red-shift of 0.15 $\mathrm{eV}$ is due to the reduction in Ga inclusion into InAs QDs from 54\% for the reference sample up to $42 \%$ for the sample with an MMHy supply of $4 \times 10^{-4} \mathrm{~Pa}$ and is also due to the additional contribution from the dot size increase. The comparison of the measurements with the other calculations assuming the $\mathrm{W} / \mathrm{H}$ ratios of 3 and 9 changed the above Ga concentration of $54 \%$ to $57 \%$ or $52 \%$ and that of $42 \%$ to $44 \%$ or $40 \%$, respectively. However, the overall characteristics showed a similar tendency to that shown in Fig. 4 and the interpretation of the PL peak red shift remained the same. The slight blue shift observed with a higher $\mathrm{N}$ supply shown in Fig. 1(b) was attributed to the reduced dot sizes (also shown in Fig. 3) slightly cancelled with the reduced Ga inclusion. Therefore the minimum of the photon energy shown in Fig. 1(b) observed at a MMHy BEP of $\sim 7 \times 10^{-4} \mathrm{~Pa}$ results from the balance of the reduced Ga inclusion and the dot size reduction, and a lower photon energy may be possible with the further optimization of the nitridation process.

The red shift may be equivalently accounted for by the $\mathrm{N}$ inclusion in the $\operatorname{In}(\mathrm{Ga})$ As dot. If the bowing parameter in $\operatorname{In}(\mathrm{Ga}) \mathrm{AsN}$ is assumed to be similar to that of GaAsN, i.e., $b=19.1$ $\mathrm{eV}^{22)} 0.8 \%$ of the $\mathrm{N}$ inclusion in the dot will result in a similar amount of the red shift. However, $\mathrm{N}$ atoms in GaAs tend to form localized states because of the large electronegativity and the much smaller atomic radius. Therefore, the $\mathrm{N}$ inclusion in QDs will broaden the PL half-width and the QD sub peaks will be less observable. These properties are opposite to the observed in this study and therefore the $\mathrm{N}$ inclusion in InAs QDs is quite unlikely. In comparison with a previous experiment on InAs QDs buried with GaAsN SCLs, ${ }^{10)}$ the present "nitridation" process supplies MMHy directly to InAs QD surfaces for $30 \mathrm{~s}$ successively for five times and gives a higher probability of $\mathrm{N}$ diffusion into the InAs QDs if the $\mathrm{N}$ diffusion is probable. However, the observed red-shift of $150 \mathrm{meV}$ in this work is much smaller than the previously observed systematic red-shifts from the photon energy range of 1.2 to $0.8 \mathrm{eV}$ in ref. 10 with the increase in $\mathrm{N}$ concentration in the GaAsN burying layers. This comparison indicates that the red-shift in the latter case is not dominated by $\mathrm{N}$ diffusion into the InAs QDs.

The possibility of the solid-phase diffusion of $\mathrm{N}$ from GaAsN SCL to InAs QDs was examined by post growth thermal annealing. What was observed was a blue shift rather than a red shift. ${ }^{10)}$ This showed that the diffusion of Ga into InAs is more enhanced than that of N. A similar “nitridation” process on InAs QD surfaces was studied using radio-frequency N plasma in MBE and a more enhanced red-shift from 1.18 to $1.033 \mathrm{eV}$ was observed. ${ }^{23)}$ This may be attributed to the active $\mathrm{N}$ radicals that enhance $\mathrm{N}$ adsorption on the surface or $\mathrm{N}$ diffusion into 
InAs QDs. In this study, the direct role of $\mathrm{N}$ in the "nitridation" process of InAs QD surfaces is apparently not evident. However, valence-force-field model calculations of stress in InAs QDs by one of the authors (Y.N.) showed that the $\mathrm{N}$ termination of the QD surfaces changed the stress direction toward the surface In atoms of the QDs. ${ }^{24)}$ This will prevent In segregation into the GaAs capping layers. Moreover, this will reduce the Ga inclusion into InAs QDs, which is consistent with the present observations.

In summary, the influence of the intentional supplies of MMHy to InAs QD surfaces just before burying with GaAs layers was studied. Emission peak wavelengths showed a red shift with an increase in MMHy supply on InAs QD surfaces but changed into a blue shift for higher MMHy supply. The red shift was caused by the reduction in Ga inclusion in QDs and the blue shift was caused by the dot size reduction as determined by AFM and TEM observations and model calculations. The discussion on the crystallographic mechanism of the dot size variations with "nitridation" needs more detailed observation of growth processes and is now under study.

This work was supported in part by a Grant-in-Aid for Scientific Research on Priority Area (A) (1), No. 17068001, from the Ministry of Education, Culture, Sports, Science and Technology, Japan. 
1) H. Zbinden, H. Bechmann-Pasquinucci, N. Gisin and G. Ribordy, Appl. Phys. B67 (1998) 743.

2) D. S. Bethune, M. Navarro and W. P. Risk, Appl. Opt. 41 (2002) 1640.

3) D. Dalacu, D. Poitras, J. Lefebvre, P. J. Poole, G. C. Aers and R. L. Williams, Appl. Phys. Lett. 84 (2004) 3235.

4) K. Takemoto, Y. Sakuma, S. Hirose, T. Usuki, N. Yokoyama, T. Miyazawa, M. Takatsu and Y. Arakawa, Jpn. J. Appl. Phys. 43 (2004) L993.

5) M. Pelton, C. Santori, J. Vuckovic, B. Zhang, G. S. Solomon, J. Plant and Y. Yamamoto, Phys. Rev. Lett. 89 (2002) 233602.

6) H. Saito, K. Nishi and S. Sugou, Appl. Phys. Lett. 73 (1998) 2742.

7) K. Nishi, H. Saito and S. Sugou, J.-S. Lee, Appl. Phys. Lett. 74 (1999) 1111.

8) J. Tatebayashi, M. Nishioka and Y. Arakawa, Appl. Phys. Lett. 78 (2001) 3469.

9) X. Q. Zhang, S. Ganapathy, H. Kumano, K. Uesugi and I. Suemune, J. Appl. Phys. 92 (2002) 6813.

10) S. Ganapathy, X. Q. Zhang, I. Suemune, K. Uesugi, B.-J. Kim and T.-Y. Seong, Jpn. J. Appl. Phys. 42 (2003) 5598.

11) X. Q. Zhang, S. Ganapathy, I. Suemune, H. Kumano, K. Uesugi, Y. Nabetani and T. Matsumoto, Appl. Phys. Lett. 83 (2003) 4524.

12) M. Sopanen, H. P. Xin and C. W. Tu, Appl. Phys. Lett. 76 (2000) 994.

13) K. Yamaguchi, Y. Saito and R. Ohtsubo, Appl. Surf. Sci. 190 (2002) 212.

14) S. K. Park, Y. J. Park, E. K. Kim, C. J. Park, H. Y. Cho, Y. S. Lim, Jeong, Y. Lee and C. Lee, Jpn. J. Appl. Phys. 41 (2002) 4378.

15) T. Tawara, S. Tanaka, H. Kumano and I. Suemune, Appl. Phys. Lett. 75 (1999) 235.

16) M. Sugawara, Phys. Rev. B 51 (1995) 10743.

17) C. G. Van de Walle, Phys. Rev. B 39 (1989) 1871.

18) M. A. Cusack, P. R. Briddon and M. Jaros, Phys. Rev. B 54 (1996) R2300.

19) I. Vurgaftman, J. R. Meyer and L. R. Ram-Mohan, J. Appl. Phys. 89 (2001) 5815.

20) G. Bester, S. Nair and A. Zunger, Phys. Rev. B 67 (2003) 161306R.

21) The energy separation of the PL subpeaks for the reference sample with no MMHy supply on the dot surfaces was estimated assuming a main Gaussian lineshape function and an additional one with a lower intensity at a higher photon energy to fit the observed asymmetric spectrum.

22) S. Sakai, Y. Ueta, and Y. Terauchi, Jpn. J. Appl. Phys. 32 (93) 4413. 
23) T. Kita, Y. Masuda, T. Mori and O. Wada, Appl. Phys. Lett. 83 (2003) 4152.

24) Y. Nabetani, T. Matsumoto, G. Sasikala and I. Suemune, J. Appl. Phys. 98 (2005) 063502.

Figure Captions

Fig. 1 I. Suemune et al
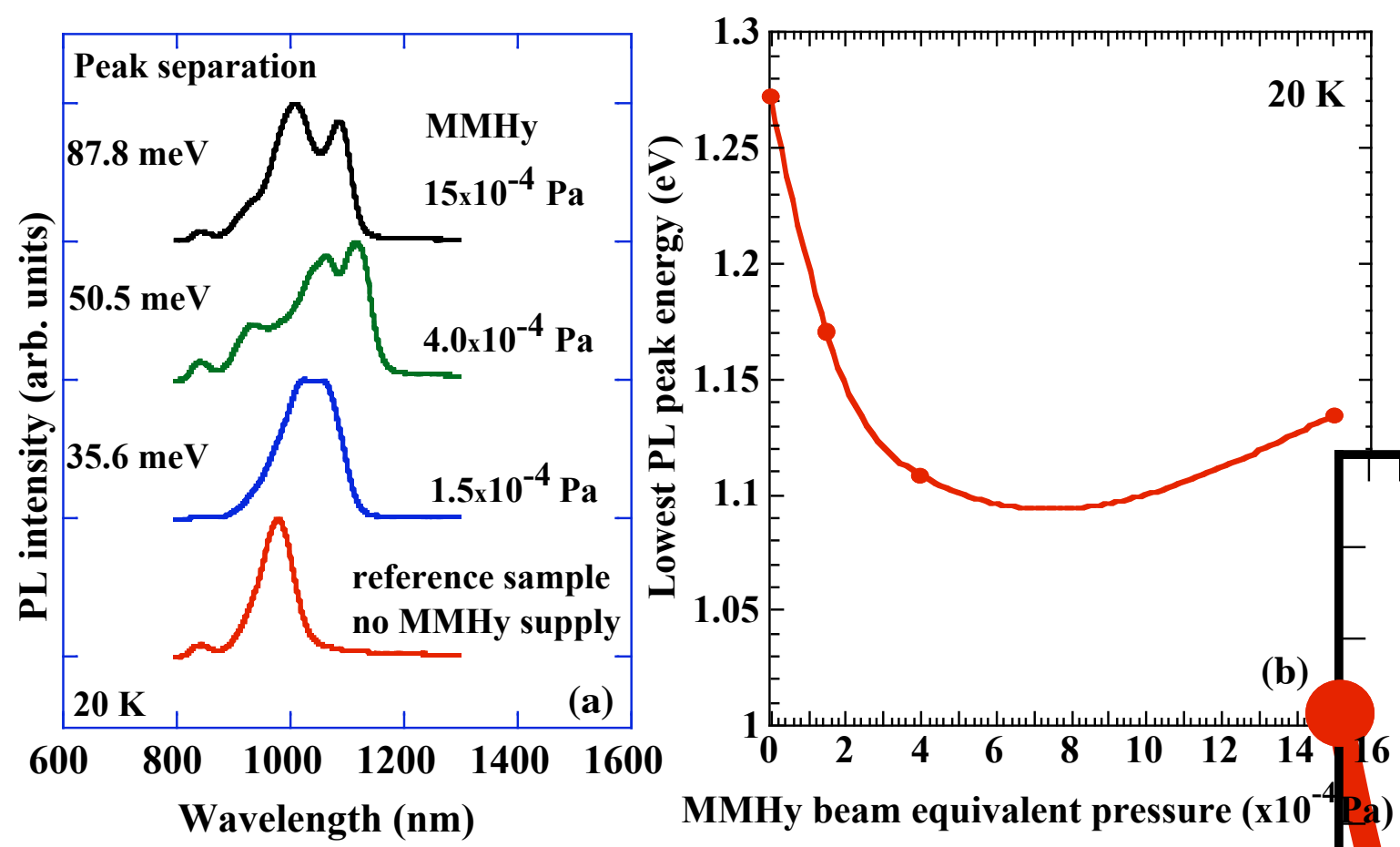

Fig. 1. (a) PL spectra of InAs QDs and their dependence on MMHy supply. (b) MMHy BEP dependence of QD lowest-state PL peak energies. 
Fig. 2 I. Suemune et al

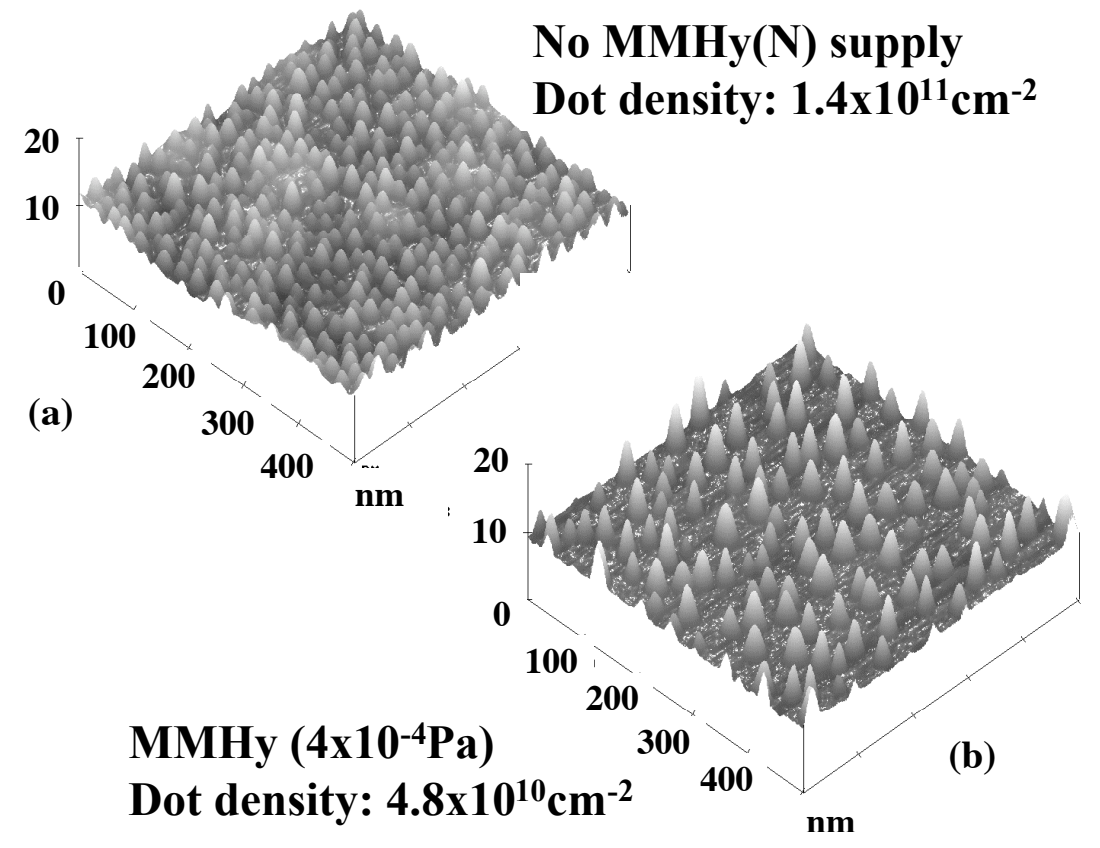

Fig. 2. AFM images of InAs QDs grown (a) without MMHy supply and (b) with MMHy supply at $4 \times 10^{-4} \mathrm{~Pa}$ on InAs QD surfaces. 
Fig. 3 I. Suemune etal

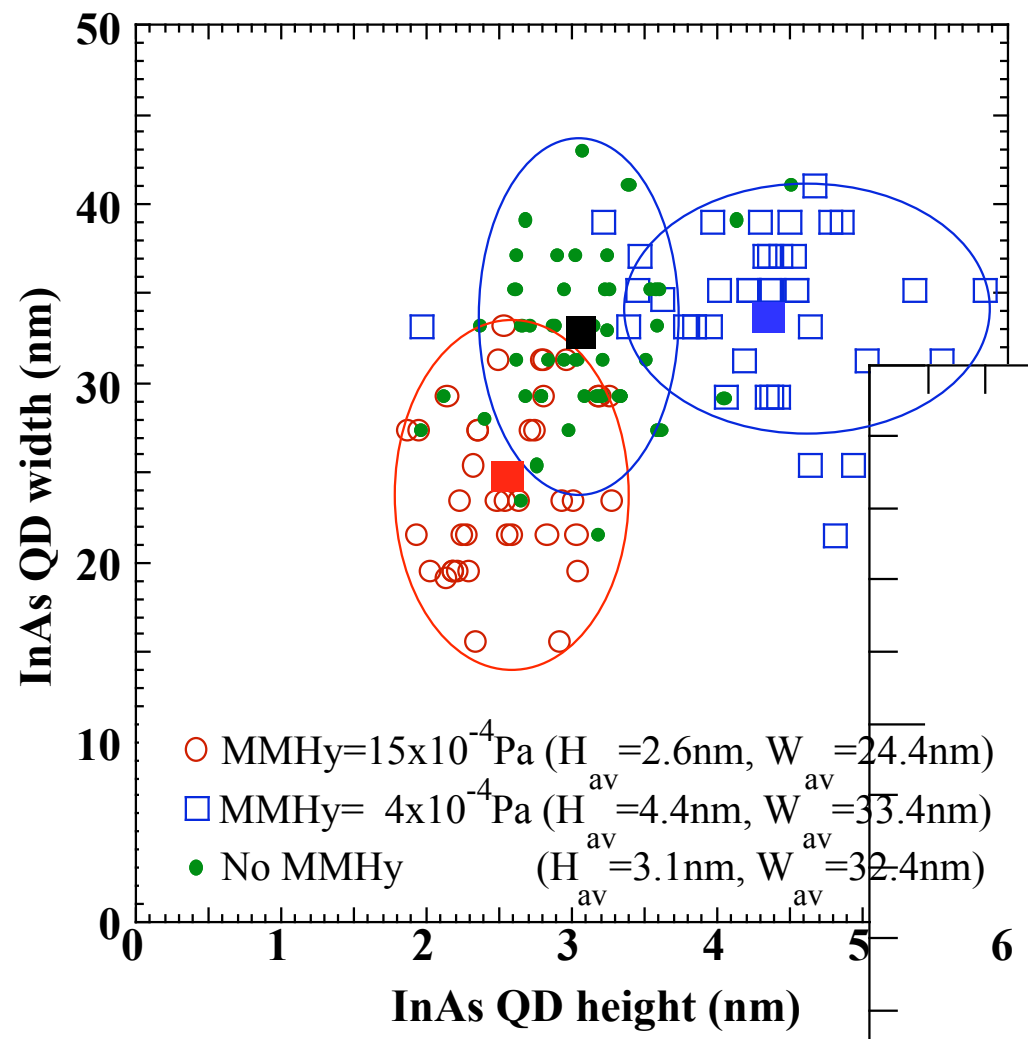

Fig. 3. InAs QD size distributions and their dependence on MMHy supply. 
Fig. 4 I. Suemune et al

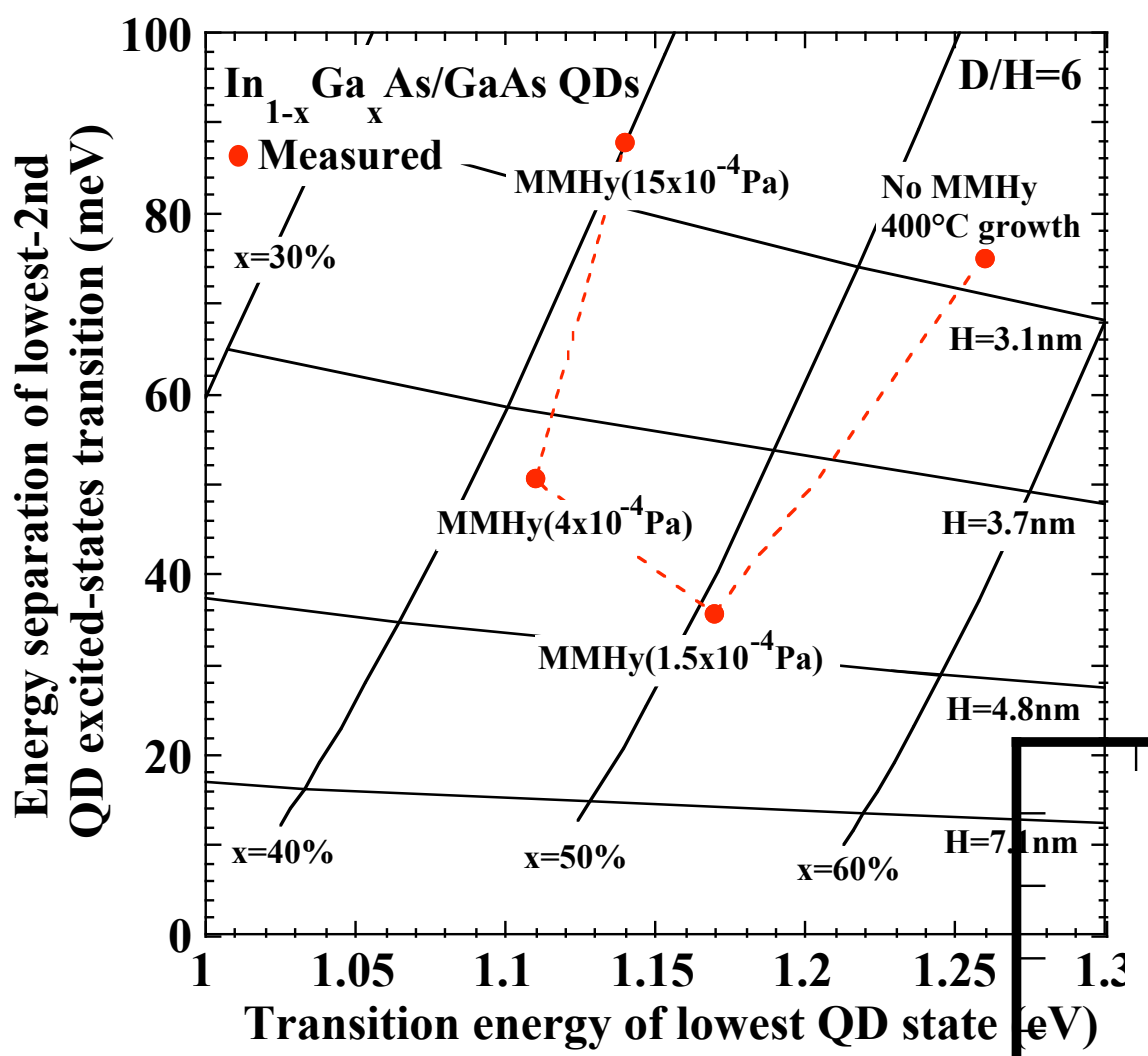

Fig. 4. Comparison of calculated relation between transition energy of lowest QD states and energy separation of lowest and second QD excited-state transitions with measurements. 